\title{
Locality Preserving Vector and Image-Specific Topic Model for Visual Recognition
}

\author{
Nguyen Anh Tu, Young-Koo Lee* \\ Department of Computer Engineering, Kyung Hee University, Korea. \\ * Corresponding author. Tel: +82-31-201-3732; email: yklee@khu.ac.kr \\ Manuscript submitted August 10, 2014; accepted January 25, 2015. \\ doi: $10.17706 /$ jcp.10.2.81-89
}

\begin{abstract}
Nowadays, evolution of mobile devices make demand for searching information increasing expressively. Many applications have been developed for recognition tasks. In this paper, we present a new and efficient visual search system for finding similar images on the large database. We first propose a compact, discriminative image representation called Locality Preserving Vector which can explicitly exploit neighborhood structure of data and attains high retrieval accuracy in the low-dimensional space. We then integrate topic modeling into visual search system for extracting topic related and image-specific information. These information enables images which likely contain the same objects to be ranked with higher similarity. The experiments show that our approach provides competitive accuracy with very low memory cost.
\end{abstract}

Key words: Object recognition, large-scale image retrieval, topic modeling.

\section{Introduction}

In recent years, multimedia and networking technologies have significantly impacted on our daily activities. Especially, development of smart phone or mobile device makes demand for searching information increasing expressively. Many applications have been developed for automatic recognition of different objects of interest, such as product catalog, landmark, art galleries... For these reasons, visual recognition and image retrieval is fascinating research field with many open challenges and opportunities. Robust visual recognition is typically achieved using scale- and rotation invariant local features (e.g, SIFT [1]).

For large-scale visual search, the bag-of-visual words (BoV) representation is widely adopted [2], since it constructs sparse frequency vector from local descriptors which can be utilized to implement efficient search via inverted list. However, BoV framework is limited when indexing in practice, since it require very high-dimensional (e.g, 1M dimensions) and sparse representation which could not apply directly efficient indexing techniques such as LSH. Also, inverted list structure can be issue for storage.

Consequently, in order to overcome above limitation and increase amount of information, higher-order representation has been proposed in Compressed Fisher Vector (CFV) [3] and VLAD [4] which included other statistic (e.g, mean and variance). Then, authors applied PCA for dimensionality reduction because original dimension of Fisher vector and VLAD representation is still high. Finally, low-dimensional representation is very compact and dense which can be employed for many efficient indexing techniques.

However, there are some important information of high-dimensional representation will not be preserved in low-dimensional space after dimension reduction step. For example, the specific of logo or name of product will be lost after projected to lower-dimension space. Then, retrieved results only show general 
information. But from viewpoint of user, the specific information is very important when they want to search information of product that they are interested in. Therefore, this issue will influence significantly to retrieval accuracy. Otherwise, dimension reduction with PCA, which is widely adopted by current wellknown frameworks [4], [5], does not explicitly consider the local neighborhood structure, since they only take into account global Euclidean structure of the data. This shortcoming of PCA will result in finding not truly similar images when we apply indexing techniques after dimension reduction step.

In this paper, we firstly develop a compact image representation called Locality Preserving Vector which can explicitly exploit and preserve neighborhood structure in low-dimensional space to be more suitable for finding similar images. Then our second contribution is to integrate topic modeling into visual search system for extracting topic related and image-specific information. These information enables images which likely contain the same objects to be ranked with higher similarity. Otherwise they further improve semantic content of image which is very necessary for visual recognition task.

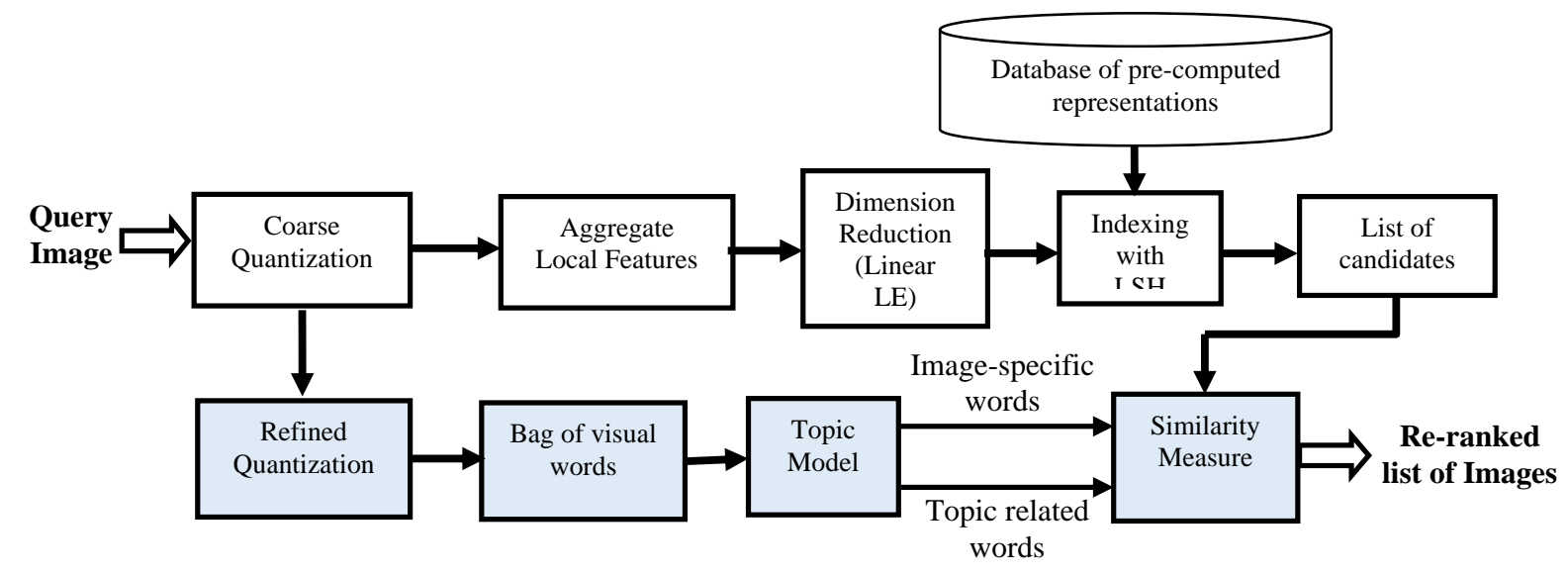

Fig. 1. Overview of proposed method.

An overview of proposed methods is illustrated in Fig. 1. Our system begins with computing LPV for image representation. Local descriptors are aggregated like VLAD and CFV framework. We then use linear LE [6] for dimension reduction to preserve neighbor-hood structure of data. Subsequently, low-dimensional representation of image can be indexed by efficient techniques (e.g., KLSH [7]). Furthermore, we use SW topic model [8] to extract topic related and image specific information which will be used to measure similarity of query image with candidate images after indexing.

\section{Locality Preserving Vector for Compact Image Representation}

As shown in general framework (Fig. 1), LPV of query image is computed and then comparing it against pre-computed database LPV for image retrieval. Similar to popular bag-of-words system, LPV can generate a ranked list accurately for large database. In opposition to BoV system, LPV obtains very compact database representation and allows much more images to be stored in memory and indexed efficiently. In this section, we will describe detailed operations of LPV framework.

\subsection{Local Descriptor Quantization}

Local descriptor quantization starts by finding regions of interest via an affine invariant detector. Then, detected regions are described using the 128-D SIFT descriptor. Similar to BoV framework, a small codebook, which is called as coarse quantizer, is learned using k-means for clustering SIFTs of database images. We obtain kc centroids which referred to coarse visual words. Then, each descriptor in an image is quantized to the nearest coarse visual word. 


\subsection{Aggregation of Local Descriptors}

After SIFT extraction and quantization, the next step in forming LPV is aggregation. Let $c_{1}, c_{2}, c_{k c}$ be a set of 128-D coarse visual words or centroids of $k_{c}$ cluster. After descriptor quantization, a set of residual vectors (vector differences between descriptors and centroids) will surround each centroids. For a given image, let $R\left(c_{i}\right)=\left\{r_{i 1}, r_{i 2}, \ldots r_{i N i}\right\}$ represents the set of $N_{i}$ residual vectors around the $i^{\text {th }}$ visual word $c_{i}$ where $r_{i j}$ $\epsilon \mathbf{R}^{128}$. Similar to VLAD, the aggregated residual for the $i^{\text {th }}$ visual is $v_{i}=\sum_{i}^{N_{i}} r_{i j}$. After that, a single vector of image is achieved by concatenation of the aggregated word residuals at all the visual words. This single vector is $\mathbf{v}=\left[\begin{array}{llll}v_{1} & v_{2} & \ldots & v_{k c}\end{array}\right] \in \mathrm{R}^{128 x k c}$.

\subsection{Dimensionality Reduction with Laplacian Eigenmaps}

Because the memory usage of the database is directly proportional to the dimension of vector $\mathrm{v}$, we want to reduce the dimension as much as possible but still guarantee for high retrieval accuracy. VLAD [4] and CFV [3] both use PCA for dimension reduction. However, PCA fail to discover the underlying structure, if data lie on a nonlinear sub-manifold of the feature space. Therefore, in our approach, we apply Laplacian Eigenmaps [9] which has locality preserving properties and very suitable for retrieval framework. This techniques has been successfully applied to, e.g. clustering[10], face recognition [11], etc.

In LE, the local properties are based on pairwise distances between near neighbor. LE computes a lowdimensional representation of data in which the distance between a datapoint and its nearest neighbors are minimized. In this paper, we use a linear variant of LE [6], because one drawback of original LF is that it cannot produce a transform function. Therefore, if the query image is not in database, we cannot map it into the dimension-reduced space.

Firstly, a neighborhood graph $\mathrm{G}$, where every vector $\mathbf{v}_{\mathbf{i}}$ is connected to its $k$ nearest neighbors, is constructed. All vertices $\mathbf{v}_{\mathbf{i}}$ and $\mathbf{v}_{\mathbf{j}}$ in graph $\mathrm{G}$ are connected by an edge, the weight of the edge is computed using the Gaussian kernel (equation 1), leading to sparse adjacency matrix $\mathbf{W}$.

$$
\mathbf{w}_{\mathrm{ij}}=e^{-\frac{\left\|\mathbf{v}_{\mathrm{i}}-\mathbf{v}_{\mathrm{j}}\right\|}{2 \sigma^{2}}}
$$

Suppose $\mathbf{p}$ is a transformation vector of linear LE, then $\mathbf{x}=\mathbf{V p}$, where the raw vectors of matrix $\mathbf{V}$ are the image representation in high-dimensional space and $\mathbf{x}$ is LPV. In computation of the low-dimensional LPV, the minimization is as below:

$$
\begin{gathered}
\underset{p}{\arg \min } \mathbf{x}^{\mathbf{T}} \mathbf{L} \mathbf{x} \\
\mathbf{x}^{\mathrm{T}} \mathbf{D} \mathbf{x}=1
\end{gathered}
$$

Now, we replace $\mathbf{x}=\mathbf{V} \mathbf{p}$ and above equations by Lagrangian formulation of the problem. The Lagrangian is as follows:

$$
\mathbf{L}=(\mathbf{V P})^{\mathrm{T}} \mathbf{L}(\mathbf{V P})-\lambda(\mathbf{V P})^{\mathrm{T}} \mathbf{D}(\mathbf{V P})
$$

The low-dimensional representation $x$ can thus be found by solving the following eigenfunction:

$$
L^{\prime} \mathbf{p}=\lambda D^{\prime} \mathbf{p}
$$

where $\mathbf{L}^{\prime}=\mathbf{V}^{\mathbf{T}} \mathbf{L V}, \mathbf{D}^{\prime}=\mathbf{V}^{\mathbf{T}} \mathbf{D V}, \mathbf{D}$ is degree matrix of $\mathbf{G}$ and the graph Laplacian $\mathbf{L}$ is computed by $\mathbf{L}=\mathbf{D}-\mathbf{W}$.

We retain $d$ most minimum eigenvector $\mathbf{p}_{\mathbf{i}}(i=1,2, \ldots d)$ corresponding to the minimum eigenvalue solutions that minimizes objective function to the generalized eigenvalue problem.

Let $\mathbf{P}=\left(\mathbf{p}_{\mathbf{1}}, \mathbf{p}_{2}, \ldots, \mathbf{p}_{\mathbf{d}}\right)$. Now, given a new point $\mathbf{v}_{\mathbf{q}}$ (e.g., query image) in the high-dimensional space, its linear mapping to the low-dimensional space can be easily computed by $\mathbf{x}_{\mathbf{q}}=\mathbf{v}_{\mathbf{q}} \mathbf{P}$. 
With linear LE, we obtain LPV which is very compact and low-dimensional representation. Our LPV is suitable for most of indexing schemes in order to obtain the high retrieval performance. In Section 4.1, we will describe more detailed how to utilize our LPV with effective indexing scheme.

\section{Image-Specific Topic Model}

As mentioned earlier, retrieving images with low-dimensional representation will easily lead to lost of specific information. Therefore we need to design a method in order to preserve this kind of information. Since image can be modeled as bag-of-words, we consider it as document. Then, there is very effective way to extract semantic and specific information by using topic models [12] which are very popular in natural language processing. Given a collection of documents, with each document represented by a BoV vector, the models are able to learn common topics such as "sports" or "movies". The models can then be used to associate relevant documents.

With the success of these models, several computer vision papers [13], [14] have applied them to the visual domain, replacing text words with visual words. Most of studies have addressed the task of learning classification or annotation, rather than retrieval and indexing which we are interested in here.

\subsection{Probabilistic Topic Model}

The first step of topic and specific-word extraction is to construct visual vocabulary. Because, number of centroids associated with coarse quantizer is small and not enough to construct reliable vocabulary for topic modeling. Therefore, we adopt refined quantization in each coarse cluster by using k-mean clustering. This size of vocabulary is now increased to $k x k_{c}$ which is more reliable to represent image as document in practice.

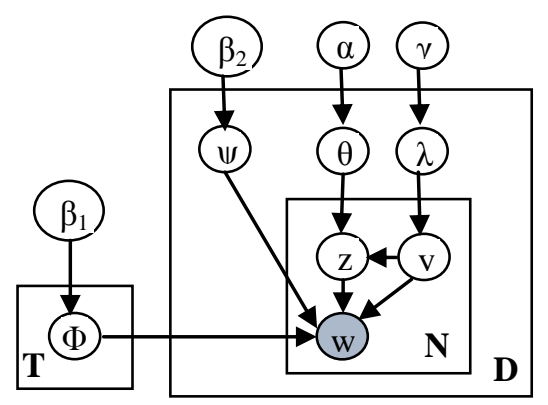

Fig. 2. Specific word topic model.

In this paper, we use special words topic model with background distribution which was proposed in [8] to identify topic and specific words. This model is an extension of well-known LDA model [12]. But in our work, we use simplified version called specific words topic model (SW) of proposed method which exclude background distribution, as shown in Fig. 2. Since, our LPV is the derivation of VLAD and FV which have been proved that can discount automatically background information of image [5]. For this reason, background information can be ignored in our topic model and result in easier implementation and competitive speed, but still maintain the same accuracy with original model.

The generative process behind is that images are represented as random mixture over latent topic and specific word distribution. SW assumes the following generative process.

1) Sample $y \sim$ mutinomial $(\lambda)$

2) For each word position $i, j$, where $j \in\left\{1,2, \ldots, N_{d}\right\}, i \in\{1,2, \ldots, D\}$

- If $y=1$ :

Sample a topic $z_{i j} \sim \operatorname{mutinomial}\left(\theta_{d}\right)$

Sample a word $w_{i j} \sim \operatorname{multinomial}\left(\Phi_{z i j}\right)$ 
- If $y=2$ :

Sample word $w_{i j} \sim \operatorname{multinomial}\left(\psi_{d}\right)$

The conditional probability of visual word $w$ given an image $d$ can be written as:

$$
\begin{aligned}
p(w \mid d)= & p(y=1 \mid d) \sum_{t=1}^{T} p(w \mid z=t) p(z=t \mid d) \\
& +p(y=2 \mid d) p^{\prime}(w \mid d)
\end{aligned}
$$

where $p^{\prime}(w / d)$ is a special word distribution for image $d$.

As above assumption, we can see that switch variable y is used to control the generation of visual word. Hence, an image contains two types of visual words where one is generated from topic distribution $\left(\Phi_{z}\right)$, the other is generated from image specific distribution $\left(\psi_{d}\right)$. The idea of SW can be interpreted that a visual word do not occur often in other images but are appeared several time in a few images will be treated as image-specific word. Otherwise, a visual word which often occur in a group of images but rarely appear in other images, will be treated as a topic related word.

\subsection{Parameter Estimation and Inference}

In this section, we describe a method for parameter estimation in SW model where we will use independent training set of $\mathrm{M}$ images. Hyperparameters can be found by maximization of following log likelihood.

$$
L\left(\alpha, \beta_{1}, \beta_{2}, \gamma\right)=\sum_{d=1}^{M} \log \left(p\left(w, y_{d}, z_{d} \mid \alpha, \beta_{1}, \beta_{2}, \gamma\right)\right)
$$

One effective way is to estimate these parameters by using EM algorithm as the following iterative process:

(E-step). We follow the collapse Gibbs sampling algorithm for joint sampling of hidden variables $z_{i}$ and $y_{i}$ for each visual word $w_{i}$. Gibbs sampling equation is as below:

$$
\begin{gathered}
p\left(y_{i}=1, z_{i}=t \mid \boldsymbol{w}, \boldsymbol{y}_{-i}, \boldsymbol{z}_{-i}, \alpha, \beta_{1}, \beta_{2}, \gamma\right) \\
\propto \frac{N_{d 1,-i}+\gamma}{N_{d,-i}+2 \gamma} \times \frac{n_{t d,-i}^{T D}+\alpha}{\sum_{t^{\prime}} n_{t^{\prime} d,-i}^{T D}+T \alpha} \times \frac{n_{w t,-i}^{W T}+\beta_{1}}{\sum_{w^{\prime}} n_{w^{\prime} t,-i}^{W T}+W \beta_{1}} \\
p\left(y_{i}=2 \mid \boldsymbol{w}, \boldsymbol{y}_{-i}, \boldsymbol{z}_{-i}, \beta_{2}, \gamma\right) \propto \frac{N_{d 2,-i}+\gamma}{N_{d,-i}+2 \gamma} \times \frac{n_{w d,-i}^{W D}+\beta_{2}}{\sum_{w^{\prime}} n_{w^{\prime} d,-i}^{W D}+W \beta_{2}}
\end{gathered}
$$

where $W$ is the size of visual vocabulary; $T$ is number of topic; the subscript $-i$ indicates that whole variables excluding the $i$ th variable; $N_{d}$ is the number of visual words in image $d$ and $N_{d 1}, N_{d 2}$ are the number of visual words in image $\mathrm{d}$ assigned to the topic related, specific words, respectively; $n_{t d}^{T D}$ is number of words assigned to topic $\mathrm{t}$ in image $d ; n_{w t}^{W T}$ is number of times word $w$ assigned to topic $\mathrm{t} ; n_{w d}^{W D}$ is number of times word $w$ assigned to image-specific words distribution of image $d$.

(M-step). We find parameter $\alpha, \beta_{1}, \beta_{2}, \gamma$ that minimize log likelihood equation (4) which is also approximate expectation and computed in E-step. These two steps are repeated until convergence.

With model parameters estimated from training images, we can infer hidden variables for database and query images. In our work, we also use collapse Gibb sampling for inference, which is similar to the estimation step, but model parameters obtained from estimation are fixed. Finally, the compound representation of an image obtained by SW model is a pair $\left\langle\theta_{d}, \psi_{d}\right\rangle$ which is computed by equation (5) from sample variables $z$. This compound representation will be used for ranking scheme as described in next 
section.

$$
\theta_{d}^{(t)}=\frac{n_{t d}^{T D}+\alpha}{N_{d 1}+T \alpha} ; \quad \psi_{d}^{(W)}=\frac{n_{w d}^{W D}+\beta_{2}}{N_{d 2}+T \beta_{2}}
$$

\section{Searching Algorithm}

As described in previous section, LPV is a discriminative and compact representation which is suitable for efficient indexing due to preserving locality in low dimension. Subsequently, SW topic model has been employed to extract topic related and image-specific visual words for ranking indexed images when querying. The overall search algorithm will be completely described in this section.

\subsection{Indexing}

Given image I with a set of extracted SIFTs. Indexing image I proceed as follows:

1) Coarse quantization for descriptors of image I: Partition descriptors into $k_{c}$ groups where each group contains descriptor vectors nearest to its centroid.

2) Aggregate local descriptors which are coarsely quantized into single vector $v$ as described in Section 2.2. Then, applying intra-normalization for vector $v$.

3) Reduce dimension of $\mathrm{v}$ by using linear LE, then we obtain LPV represented by vector $x$.

4) For each group of descriptors in step 1, splitting them into $k$ smaller groups by refined quantization. Hence, image I can be represented as BoWs $\left[t_{1}, t_{2}, \ldots, t_{w}\right]$ where W is size of vocabulary ( $\left.\mathrm{W}=k \mathrm{x} k_{c}\right), t_{i}$ is occurrences of word $i$ assigned to image I.

5) BoW representation of image I is applied to SW model to extract image-specific and topic related information. Then, compound representation of image is $\left\langle\theta_{d}, \psi_{d}\right\rangle$.

6) Index image I with LPV representation by using LSH. In this paper, we choose KLSH which is unstructured LSH and has been shown that outperform with other LSH scheme [7]. Subsequently, compound representation $<\theta_{d}, \psi_{d}>$ is stored as semantic information of indexed image I.

\subsection{Searching}

Searching the nearest neighbor of query image $I_{q}$ consist of:

1) Compute low-dimensional representation LPV of query image.

2) Retrieve an initial list of candidate images from database corresponding to $I_{q}$ via $\mathrm{KLSH}$

3) Compute similarity of query image to each image in list of candidate images as following equation:

$$
\operatorname{Sim}\left(I_{q}, I_{d}\right)=\rho_{1} \cos \left(\theta_{q}, \theta_{d}\right)+\rho_{2} \cos \left(\psi_{q}, \psi_{d}\right)
$$

where $\rho_{1}, \rho_{2}$ are topic related and image-specific proportion; $\left\langle\theta_{d}, \psi_{d}\right\rangle$ and $\left\langle\theta_{q}, \psi_{q}\right\rangle$ are compound representation of database and query image, respectively.

4) Select $k$ nearest neighbor of query image based on similarity score computed in above step.

Due to property of locality preserving of low dimensional representation, we can achieve a reliable candidate set in step 2 . Then, images are re-ranked via semantic information. In experiment, we will show better performance of proposed method in comparison with other approaches.

\section{Experiments}

\subsection{Data Sets and Experimental Setting}

In this paper, we use publicly available dataset to evaluate our performance and compare to existing methods.

Dataset: All results are reported on two standard datasets: UKB [15] and Inria Holiday[16]. The UKB dataset contains 10200 images of 2550 objects, each of which is represented by 4 images. The Inria 
Holidays dataset contains 1491 images of 500 groups, each of which represents a scene or object. The retrieval accuracy is evaluated by mean Average Precision (mAP) over 500 queries for Holidays dataset and 2550 queries for UKB dataset. For training, we used independent dataset which is Flickr60k. This dataset contains 60k images captured from Flickr and made available at [16].

Experimental setting: Local SIFTs are extracted as procedure in [1]. Then, we use Flickr 60k to train the coarse and refined vocabularies. Flickr60k are also used to learn linear LE and parameters of SW model. Our approach is compared to two well-known methods which image represented by BoVs [17] and Fisher vector [4].

\subsection{Performance Comparison without Ranking Scheme}

Fig. 3 shows performance comparison of our method without ranking scheme and well-known VLAD and Fisher. Here, we emphasize on the changing of performance with respect to different dimensions. Number of coarse centroids $k_{c}$ of LPV is set to 100 . It also corresponds to the number of centroids for VLAD, and to the number of mixture components for Fisher vector when aggregating local SIFT descriptors. All methods use KLSH for indexing.

Due to higher-order statistics and complexity, Fisher vector gives slightly better accuracy than VLAD and LPV at original dimension $(D=128 \times 100)$. However, when dimension reduction is applied, performance of VLAD and Fisher with PCA is decreased much faster than our LPV on both UKB and Holiday dataset. The difference of performance between very low dimension (e.g., 50) and original dimension of LPV is not significant. Otherwise, LPV performs much better than VLAD and Fisher with PCA at the same low dimension. This observation shows that our LPV are especially suitable for the case where big dimensionality reduction needs to be performed.
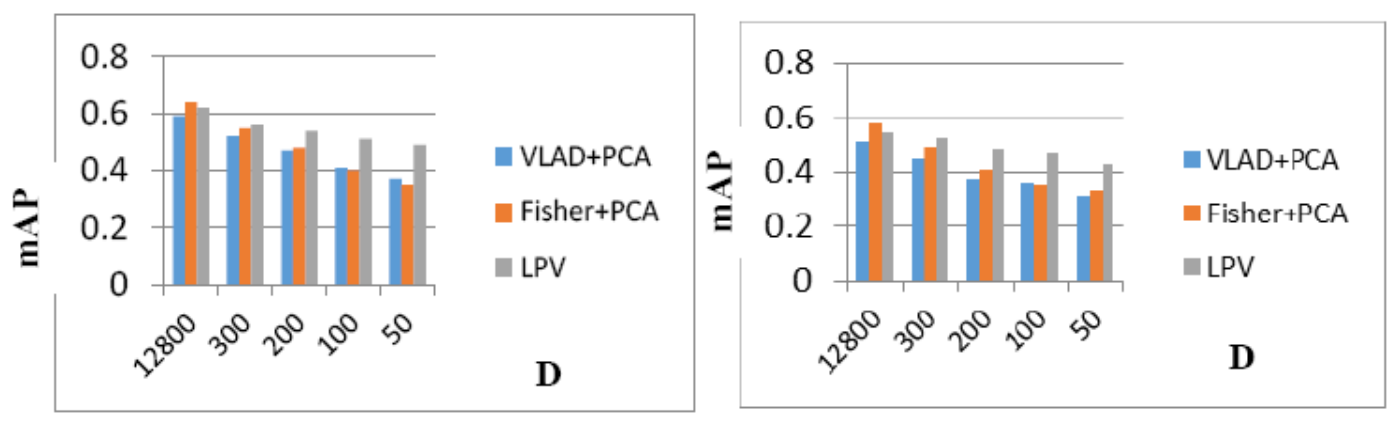

Fig. 3. Performance comparison of VLAD, Fisher and LPV.

Top: Inria Holiday Dataset. Bottom: UKB Dataset

\subsection{Performance Comparison with Ranking Scheme}

We compared our method with ranking scheme as described in Section 4 with Fisher representation and BoV model [17]. In order to show superiority of proposed method, we use Fisher without dimension reduction which usually provides best accuracy. In our approach, we constructed vocabulary with $50 \mathrm{k}$ visual words for topic modeling. This vocabulary is also used to compute BoV representation. In our experiment, we set number of topics to 80 . Otherwise, we choose 50 specifics word for each image, which correspond to largest values of $\psi$ components, and construct compound representation $\langle\theta, \psi\rangle$ for similarity measure.

The results are given in Table 1 . We observed that our method outperforms significantly Fisher and BoV model, even though it require much smaller space for representation $(D \approx 200)$ compared to huge dimension of BoV $(D=50 \mathrm{k})$ and Fisher vector $(D=12 \mathrm{k})$. This is the reason why our proposed method, which combines 
low-dimensional representation with semantic information (e.g., specific and topic-related words), are more general to practical applications.

Table 1. Performance Comparisons for Proposed Method with Ranking Scheme and Well-Known Methods

\begin{tabular}{|l|c|c|}
\hline \multicolumn{1}{|c|}{ Method } & Inria Holidays & UKB \\
\hline BoV & 0.61 & 0.65 \\
Full Fisher & 0.64 & 0.59 \\
LPV with ranking & 0.73 & 0.71 \\
scheme & & \\
\hline
\end{tabular}

\section{Conclusion}

In this paper, we have presented Locality Preserving Vector (LPV) which is a compact and lowdimensional image representation. LPV outperforms existing methods such as VLAD and Fisher vector under different dimension. It shows that our LPV can be used effectively for storing large database on memory. Furthermore, we integrate SW model into retrieval system to preserve specific and related-topic information and use them for similarity measure in ranking scheme. LPV with ranking scheme show significant improvement of accuracy compared to well-known methods (e.g., BoV and Fisher).

\section{Acknowledgement}

This research was supported by the MSIP (Ministry of Science, ICT\&Future Planning), Korea, under the ITRC (Information Technology Research Center) support program (NIPA-2014(H0301-14-1020)) supervised by the NIPA (National IT Industry Promotion Agency).

\section{References}

[1] Lowe, D. G. (2004). Distinctive image features from scale-invariant key- points. International Journal of Computer Vision, 60, 91-110.

[2] Sivic, J., \& Zisserman, A. (2003). Video Google: A text retrieval approach to object matching in videos. Proceedings of IEEE International Conference on Computer Vision.

[3] Perronnin, F., Liu, Y., Sánchez, J., \& Poirier, H. (2010). Large-scale image retrieval with compressed fisher vectors. Proceedings of IEEE Conference on Computer Vision and Pattern Recognition.

[4] Jégou, H., Douze, M., Schmid, C., \& Pérez, P. (2010). Aggregating local descriptors into a compact image representation. Proceedings of IEEE Conference on Computer Vision and Pattern Recognition.

[5] Jégou, H., Perronnin, F., Douze, M., Sánchez, J., Pérez, P., \& Schmid, C. (2012). Aggregating local image descriptors into compact codes. IEEE Transactions on Pattern Analysis and Machine Intelligence, 34, 1704-1716.

[6] He, X., \& Niypgi, P. (2004). Locality preserving projections. Advances in Neural Information Processing Systems.

[7] Paulevé, L., Jégou, H., \& Amsaleg, L. (2010). Locality sensitive hashing: A comparison of hash function types and querying mechanism. Journal of Pattern Recognition Letters, 31, 1348-1358.

[8] Chemudugunta, C., Smyth, P., \& Steyvers, M. (2006). Modeling general and specific aspects of documents with a probabilistic topic model. Advances in Neural Information Processing Systems.

[9] Belkin, M., \& Niyogi, P. (2004). Laplacian Eigenmaps and spectral techniques for embedding and clustering. Advances in Neural Information Processing Systems.

[10] Park, J. H., Zhang, Z., Zha, H., \& Kasturi, R. (2004). Local smoothing for manifold learning. Proceedings of IEEE Conference on Computer Vision and Pattern Recognition. 
[11] He, X., Yan, S., Hu, Y., Niyogi, P., \& Zhang, H. J. (2005). Face recognition using laplacianfaces. IEEE Transactions on Pattern Analysis and Machine Intelligence, 27, 328-340.

[12] Blei, D., Ng, A., \& Jordan, M. (2003). Latent dirichlet allocation. Journal of Machine Learning Research.

[13] Wang, C., Blei, D., et al. (2009). Simultaneous image classification and annotation. Proceedings of IEEE Conference on Computer Vision and Pattern Recognition.

[14] Li, L. J., Socher, R., et al. (2009). Towards total scene understanding: Classification, annotation and segmentation in an automatic framework. Proceedings of IEEE Conference on Computer Vision and Pattern Recognition.

[15] Nister, D., \& Stewenius, H. (2006). Scalable recognition with a vocabulary tree. Proceedings of IEEE Conference on Computer Vision and Pattern Recognition.

[16] Jégou, H., Douze, M., \& Schmid, C. (2008). Hamming embedding and weak geometric consistency for large scale image search. Proceedings of European Conference on Computer Vision.

[17] Philbin, J., Chum, O., Isard, M., Sivic, J., \& Zisserman, A. (2007). Object retrieval with large vocabularies and fast spatial matching. Proceedings of IEEE Conference on Computer Vision and Pattern Recognition.

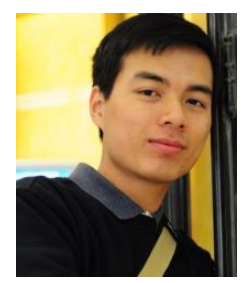

Nguyen Anh Tu received his B.S. degree from Ho Chi Minh University of Technology in 2010. Currently, he is working toward his Ph.D degree in the Department of Computer Engineering, Kyung Hee University, South Korea. His research interests include computer vision, machine learning and data mining.

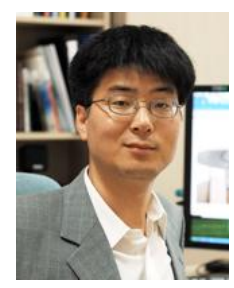

Young-Koo Lee got his B.S., M.S. and Ph.D. degrees in computer science from Korea advanced Institute of Science and Technology, Korea. He is a professor in the Department of Computer Engineering at Kyung Hee University, Korea. His research interests include ubiquitous data management, data mining, and databases. He is a member of the IEEE, the IEEE Computer Society, and the ACM. 